\title{
Neural Stem Cells Therapy to Treat Neurodegenerative Diseases
}

\author{
Weibai Chen ${ }^{1}$ \\ ${ }^{1}$ Department of life sciences, Guangzhou Medical University, Guangzhou, Guangdong, 511436, China.
}

\begin{abstract}
Neural stem cells have the ability to proliferation, differentiate and renew, which plays an important role in the growth, maturation and senescence of the human brain. But according to researches, neural stem cells in the brain do not remain active throughout an organism's lifetime. Many neural stem cells become dormant when the brain matures, and may be activated when the body is sick to selectively heal the disease. In recent years, there are many studies on neural stem cells. Joshua[1] and Ting Zhang[2] show that neurodegenerative diseases such as ischemic stroke, Alzheimer's disease and Parkinson's disease can be improved by the transplantation of neural stem cells, however the specific mechanism is not clear. This paper investigates three main questions: Why neural stem cell transplantation is chosen as a treatment? Where does NSCs derive from in clinical transplantation? How does neural stem cell transplantation treat brain diseases? And we also figure out the answers to these three questions. Firstly, transplantation of hypothalamic NSCs can delay the process of aging in the host, and Chemokines and EVs which secreted by neural stem cells can delay aging and defend neurodegenerative diseases. Secondly, the sources of NSCs can be divided into three types. The first is to isolate NSCs from primary tissue and cultivate them in vitro. The second is to produce the required cells by inducing pluripotent stem cells and embryonic stem cells. The third way to get NCS is through transdifferentiation of somatic cells. Thirdly, in brain diseases, transplanted NSCs can migrate from the aggregation site to the site of the disease, reducing damage to the blood-brain barrier, repairing learning and memory abilities that depend on the hippocampus and secreting neurotrophic factors.
\end{abstract}

\section{Introduction}

Neurodegenerative diseases are difficult to treat and they can lead to loss of motor function, memory and are even life threatening. Traditional treatments can delay the progress of the disease; however, it cannot restore nerve function or regenerate nerves. Recent studies have shown that neural stem cells play a role in cell regeneration and repairing injuries in diseases related to the nervous system [5]. Neural stem cells (NSCS), also known as pluripotency, are cells with the ability of renewal and multidirectional differentiation. These stem cells are present throughout a person's life, but studies suggest that they are mostly dormant in adult [3]. These properties of neural stem cells suggest that self-healing after brain damage is less likely. By reviewing some existing literature, three questions are going to be figured out: Why neural stem cell transplantation is chosen as a treatment? Where do NSCs derive from in clinical transplantation? How does neural stem cell transplantation treat brain diseases? One element of NSC therapy for brain injury is that NSCs can migrate from the aggregation site to the injury site under certain conditions, whereas some studies have shown that brain damage can inhibit the normal migration of NSCs [4]. With this article, the author aims to make a summary of the ideas of neural stem cells in the treatment of neurodegenerative diseases and help people understand the mechanism of neural stem cells on the nervous system in combination.

\section{Proliferation and disease}

\subsection{Why neural stem cell transplantation is chosen as a treatment?}

Human NSCs express many chemokines and chemokine receptors, which are important for migration in the brain. In mammalian brains, NSCs may still be attracted, even if they are far from the regions of neurodegeneration. Interestingly, NSCs can also produce inflammatory chemokines, which may cause neurodegenerations [13].

Webb (2018) [6] and Sun (2019) [7] have shown that extracellular vehicles (EVs) that derived from NSCs in the hypothalamus have therapeutic significance for brain injuries. EVs are vesicular corpuscle that fall off from cell membranes or are secreted by cells. They are widely found in body fluids, carrying various biological molecules, such as miRNA and protein. EVs are important tools for cells to achieve physiological functions. The figure1 below illustrates the various functions of EVs. NCS-EVs have many functions. In the nervous system, NSC-EVs have been proved to encourage neuroprotection in stroke mice and stroke pigs. 
During brain development, NSC-EVs can act as microglial morphogens to regulate the activity of microglia. In addition, NSC-EVs can control the aging process through specific EVs released by the hypothalamus [14].

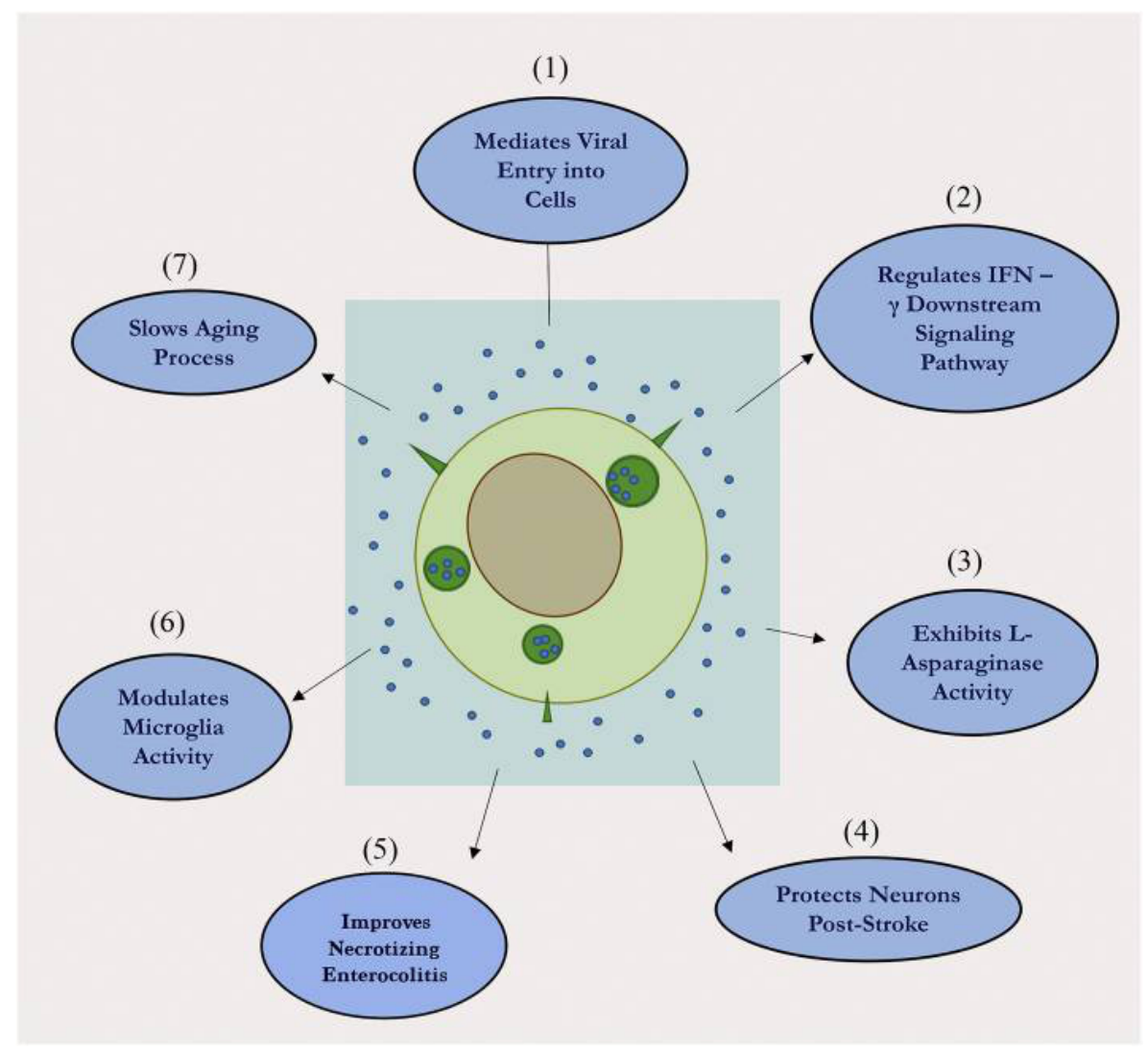

Fig. 1 The variety of properties and functions of NSC-EVs

This suggests that the aging and degenerative diseases of the brain may be affected by EVs. If NSCs disappear in the later life of the mice, it will speed up the process of aging. The disappearance of NSCs does not affect the surrounding of hypothalamic neurons, while it can lead to a number of age-related changes, which possibly lead to some diseases. Studies have shown that allogeneic transplantation of hypothalamic NSCs can delay the process of aging in the host. However, interestingly, transplanting other kinds of stem cells such as astrocytes and mesenchymal stem cells do not benefit aging. This illustrates the unsubstitutability of NSC transplantation in the treatment of neurodegenerative diseases. However, the proportion of NSCs in the hypothalamus decreases with age, meanwhile, the content of the miRNA from EVs also decreases significantly, which can lead to senescence. In the study, the healthy hypothalamus NSCs were transplanted into the brains of aging mice, releasing EVs that carry certain miRNA. There will be a corresponding decrease in neurodegenerative symptoms in the transplanted mice [14].

Chemokines and EVs are secreted by neural stem cells, which can delay aging and fight neurodegenerative diseases. In turn, neurodegenerative diseases may also influence the generation of NSCs. In the adult brain, the production of new neurons is regulated by the network of neurons, which are connected with the movement and learning functions. It suggests that the signals, which regulate the production of new neurons may be enhanced by the needs of the movement or learning. This means that if the need for these increases, activated neural stem cell regions may produce new neurons more efficiently. It can be speculated that when people suffer from brain diseases that lead to decreased learning and motor ability, it may also reverse the effect of neural stem cells which to produce new neurons more efficiently [3].

Based on existing studies, the Hippo pathway may regulate NSCs. NSCs control their mitotic activities to decide whether to proliferate or remain inactive. In the late embryonic stage of drosophila's larvae, NSCs enter a resting area, and this progress is regulated by intrinsic transcription factors. This suggests that there are only a limited number of NSCs that will stay active in adult Drosophila. This can be the reason that most adult neural stem cells are dormant. Cellular-contact inhibition through the classical Hippo signaling pathway causes neural stem cells to remain stationary. Hippo pathway activity is mediated by two intercellular transmembrane proteins, namely Crumb and Echinoid. With both of them, neural stem cells will keep quiescent.

At the same time, glial cells secrete a glycoprotein that keeps NSCs at rest. They make NSCs more easily to be stationary. Studies have shown that Salvador laurel/Hippo/Warts (SHW) signalling pathway control the flies and vertebrate cells proliferation. Crumb and 
Echinoid activate the SHW pathway, keeping NSCs stationary and inhibiting their proliferation. Many SHW regulators are known. In order to study how external signals, regulate SHW's intrinsic cell growth inhibitory activity, Ding and his team tested the transmembrane protein Crumbs and Echinoid, and their role in inhibiting NSC growth. In figure 2, through RNAi, when targeting crb or ed in NSCs, it can be measured that the size of both groups of NSC increased significantly compared to the control group (Figure 2a).
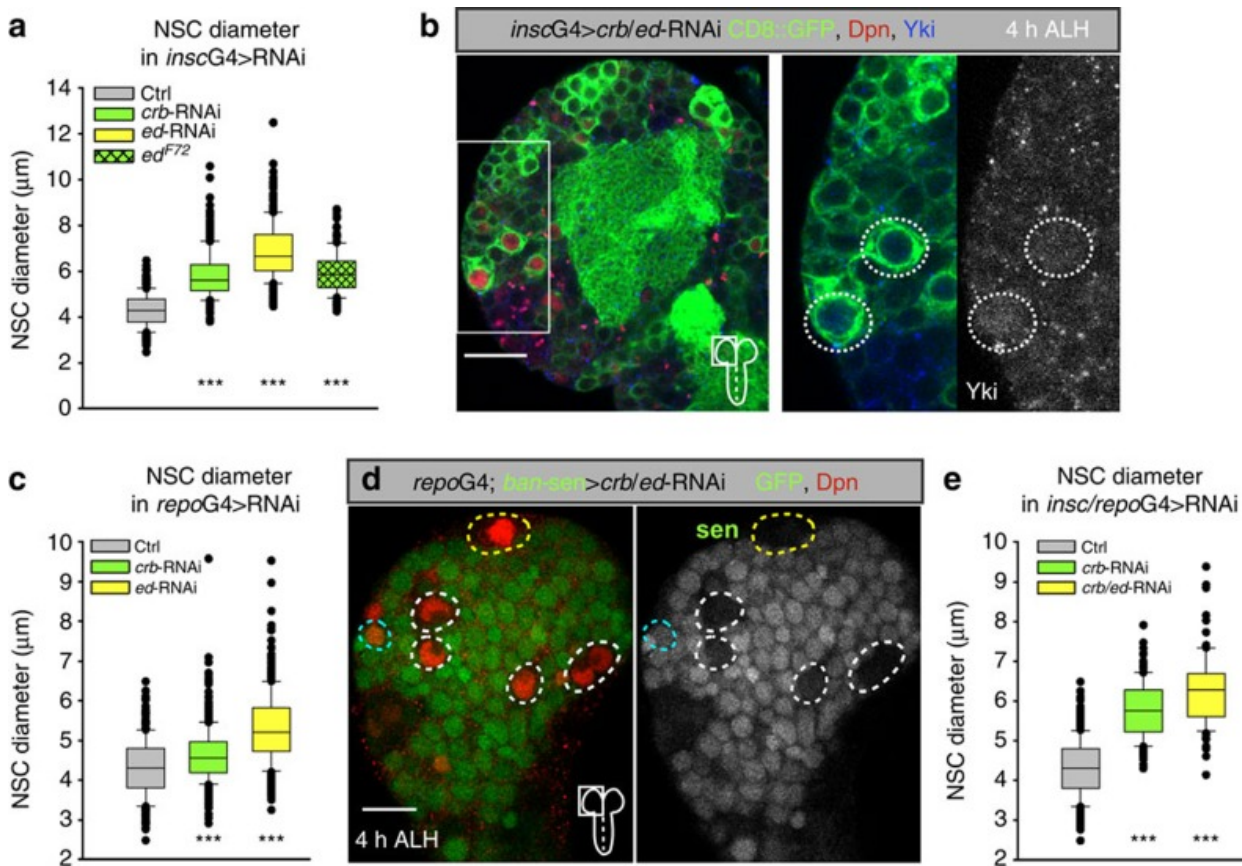

Fig. 2 NSCs become larger when targeting crb or ed in NSCs or glial cells

Meanwhile, since the reactivation of neural stem cells involves the activity of niche glia cells, we explored the signal effects of niche glia cells during quiescent. We only conducted RNA interference on crb or ed in glial cells, which was enough to observe that the NSC size of the experimental group increased significantly compared with the control group (Figure 2c) [8]. From this phenotype of NSCs, we can conclude that the two proteins Crumbs and Echinoid play a key role in affecting the growth of NSCs. At the same time, we can also conclude that the presence of these two proteins is very important for the activation of the SHW pathway. If Crumbs and Echinoid are present, they will activate the SHW pathway, thereby inhibiting the growth of NSCs. If there are a way to knock out the two proteins that control the Hippo pathway, it might be possible to put more NSCs into the treatment of disease. This might be a new way for endogenous stem cells to treat neurodegenerative diseases. However, a suitable method about the Hippo pathway has not been found. In addition, NSCs transplantation is still the main treatment for neurodegenerative diseases.

\subsection{Where do NSCs derive from in clinical transplantation?}

Cell origin is a problem that must be addressed in the treatment of neurodegenerative diseases by NSC transplantation, because the high concentration of NSCs is needed for the successful treatment of diseases. The sources of NSCs can be divided into three types. The first is to isolate NSCs from primary tissue and cultivate them in vitro, which can provide abundant cells. At the same time, epidermal growth factor and basic fibroblast growth factor together can induce the proliferation of NSCs in vitro. The second method is to produce the required cells by inducing pluripotent stem cells and embryonic stem cells, this method can help neural differentiation. Another way to get NCS is through transdifferentiation of somatic cells [15].

\subsection{How neural stem cell transplantation treats brain diseases?}

\subsubsection{Ischemic Stroke}

Stroke can increase the production of new neurons in the infarcted areas of the cerebral cortex and striatum. These cells are derived from neural stem cells in the SVZ zone. And stroke seems to provide them with an induction signal that make some asymmetric division of NSCs become symmetrical division. This can increase the number of NSCs, and make it faster to produce new neurons. Despite the body's healing way, the number of new neurons in the cortex and striatum is still low in stroke patients. We illustrate that many new cells are eliminated. Injury to the blood-brain barrier, reperfusion injury, and infiltration of immune cells lead to a deeper damage to nerve tissue in the early stage of stroke. Furthermore, neural stem cell transplantation has been shown to reduce such brain damages. For example, mice transplanted with human NSCs showed the decreased expression of inflammatory factors and decreased 
activation of microglia. What is more, transplanted NSCs migrate from the aggregation site to the site of the disease, thus reducing damage to the blood-brain barrier, suggesting that NSCs have anti-inflammatory effects. When stroke happens, immune cells enter the brain parenchyma through the impaired blood-brain barrier, and NSCs transplantation reduces central nervous injury by suppressing inflammatory cascades during the subacute phase of cerebral infarction. Clinical studies have shown that NSCs can differentiate into mature neurons after transplantation. Moreover, the mature neurons can form complete neural circuits with implanted neurons, which is of great significance to the improvement of patients' neural functions [5].

\subsubsection{Alzheimer's disease}

Alzheimer's disease is a neurodegenerative disease that is very common in the elderly. Notably, decreased expression of neurotrophic factors and reduced neuronal transport neurotrophic factors are found in Alzheimer's disease and can lead to significant loss of synapses and neurons. Marsh have shown that transplantation of mouse-derived NSCs into the hippocampus of diseased mice can repair learning and memory abilities that depend on the hippocampus. Moreover, the expression level of brain-derived neurotrophic factors increases in the brain implanted into neural stem cells, and a large amount of brain-derived neurotrophic factor can also be produced by neural stem cells in vitro [9].

\subsubsection{Parkinson's disease}

Parkinson's disease is a neurodegenerative disease caused by the loss of cells in the substantia nigra of the midbrain. Despite drug treatments may improve symptoms, it does not cure the disease [12]. Parkinson's disease and Alzheimer's disease are similar in that neurotrophic factors are lost in important areas of the brain. Besides, brain-derived neurotrophic factors and glial neurotrophic factors are also associated with the development and function of dopamine in the midbrain. Several studies have shown that neural stem cells that overexpress glial neurotrophic factor may be an effective treatment for the disease. The research of Marsh et al. indicates that the most significant repair after NSC transplantation in preclinical models may come from neurotrophic factors secreted by neural stem cells [9].

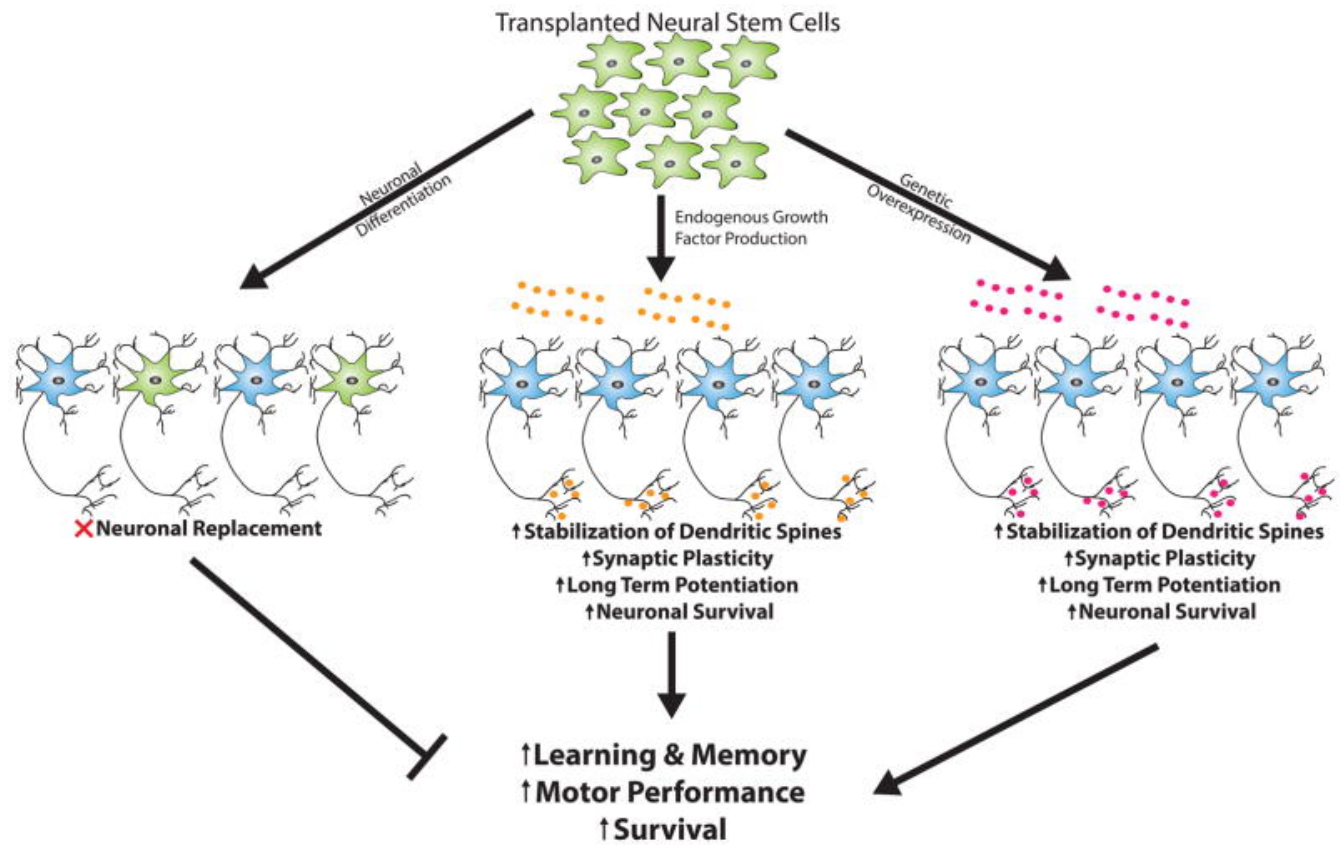

Fig. 3 Potential mechanisms of neurotrophic support provided by transplanted neural stem cells (NSCs)

At the beginning, the researchers tried to use NSC to replace neurons, inferring that after neuron differentiation (neurons; green), neuronal cells will integrate with primary neuron circuits (blue) to replace various kinds of neurons. Another path, spontaneous secretion of neurotrophic factors like BDNF, GDNF, IGF-1 and NGF (center, orange), or gene overexpression (right, purple) may offer an alternative to NSC-based treatments, perhaps A more approachable. The transplanted neural stem cells can secrete neurotrophies, augment synaptic plasticity, enhance long-term enhancement ability and promote neuron survival, and jointly improve cognitive ability and athletic performance. In addition, other studies have found that mouse neural stem cells overexpressing GDNF can prevent the degeneration of dopamine neurons in the substantial nigra [9].

\section{Discussion}

Although replacement of damaged neurons has been the primary target of neural stem cell transplantation, Lu (2013) [10] and $\mathrm{Lu}$ (2003) [11] have shown that neurotrophic factors produced by neural stem cell transplantation are also the primary factor that reduces brain damage. 
Initially, the goal of transplanting NSCs was to replace dead neurons in the host, and to connect and integrate with the original neural circuits of the host, after the transplanted stem cells differentiated. This direction was proved to be very challenging after the experiments, so people turned their attention to the neurotrophic cells. The transplantation of NSCs into the host endogenously secrete neurotrophic factors and overexpress gene can promote neuronal survival and enhance synaptic plasticity. It can improve the ability of learning and memory as well as sports performance, these functions support that neurotrophic factors play a role in the treatment of neurodegenerative diseases. However, when neurotrophic factors administration is given directly through the periphery of brain, neurotrophic factors cannot penetrate the blood-brain barrier, and the direct injection of neurotrophic factors also shows many defects. Hence, the focus has been shifted to stem cells. Nevertheless, in the new study, NSCs change its status to a potential carrier of neurotrophic factors and improve clinical outcomes [9].

\section{Conclusion}

The reasons for choosing neural stem cell transplantation as a treatment method are as follows, it can secrete chemokines and contain a certain kind of miRNA that shows strong links to brain development and disease, and it can secrete extracellular vehicles (EVs) that have therapeutic significance for brain injuries. Recent studies have suggested that neural stem cell transplantation can replace damaged neurons to form complete neural circuits in the brain. However, the specific mechanism is still uncertain and needs to be proved by subsequent studies. NSCs in the brain show the dormant state after maturity, and the Hippo pathway that controls the status of NSCs may provide follow-up study a train of thought. By genetic modification or protein knockout method, the endogenous neural stem cells may be activated, and can largely correct differentiation of neurons as people need. According to research, there are three sources of NSCs. One is the isolation of NSCs from primary tissues. The second is to generate the required cells by inducing pluripotent stem cells and embryonic stem cells. The third is to obtain neural stem cells through transdifferentiation of somatic cells. It has been shown that neural stem cell transplantation can reduce brain damages. Clinical studies have shown that NSCs can differentiate into mature neurons after transplantation. In the preclinical model, the most important repair after NSC transplantation may come from neurotrophic factors secreted by neural stem cells. On the other hand, how to make the NSCs transplanted into the host brain to replace the damaged neurons and form complete neural circuits is also worth studying.

\section{References}

1. D. Joshua, L. Bernstock, D. Peruzzotti-Jametti, et al. Neural stem cell transplantation in ischemic stroke: a role for preconditioning and cellular engineering.
Journal of cerebral blood flow and metabolism: official journal of the International Society of Cerebral Blood Flow and Metabolism. 2017.

2. T. Zhang, W. Ke, X, Zhou, Y. Qian, S. Feng, \& R. Wang, et al. Human neural stem cells reinforce hippocampal synaptic network and rescue cognitive deficits in a mouse model of alzheimer's disease-sciencedirect. 2019.

3. P.A. Carpentier, \& T.D. Palmer. Immune influence on adult neural stem cell regulation and function. Neuron, 2009, 64(1), 79-92.

4. K. Obora, Y. Onodera, T. Takehara, J. Frampton, J. Hasei \& T. Ozaki, et al. Inflammation-induced mirna-155 inhibits self-renewal of neural stem cells via suppression of ccaat/enhancer binding protein $\beta$ (c/ebp $\beta$ ) expression. 2017, rep, 7, 43604.

5. L. Wang. Research progress of neural stem cell therapy in the treatment of ischemic stroke. Journal of Shenyang Medical College, 2019,21 (05): 469-474.

6. R.L. Webb, E.E. Kaiser, B.J. Jurgielewicz, S. Spellicy, S.L. Scoville \& T.A. Thompson, et al. Human neural stem cell extracellular vesicles improve recovery in a porcine model of ischemic stroke. Stroke, 2018, STROKEAHA.117.020353.

7. M.K. Sun, A.P. Passaro, C.F. Latchoumane, S.E. Spellicy \& L. Karumbaiah. Extracellular vesicles mediate neuroprotection and functional recovery after traumatic brain injury. Journal of Neurotrauma, 2019, 37(11).

8. R. Ding, K. Weynans, T. Bossing, C.S. Barros \& C. Berger. The Hippo signaling pathway maintains quiescence in Drosophila neural stem cells. Nature communications, 2016, 7, 10510.

9. S.E. Marsh \& M. Blurton-Jones. Neural stem cell therapy for neurodegenerative disorders: the role of neurotrophic support. Neurochemistry International, 2017, S019701861730102X.

10. B. Lu, G. Nagappan, X. Guan, P.J. Nathan \& P. Wren. Bdnf-based synaptic repair as a disease-modifying strategy for neurodegenerative diseases. Nature Reviews Neuroscience, 2013, 14(6), 401.

11. P. Lu, L.L. Jones, E.Y. Snyder \& M.H. Tuszynski. Neural stem cells constitutively secrete neurotrophic factors and promote extensive host axonal growth after spinal cord injury. Experimental Neurology, 2003, 181(2), 115-129.

12. D.H. Choi, J.H. Kim, S.M. Kim, K. Kang, D.W. Han \& J. Lee. Therapeutic Potential of Induced Neural Stem Cells for Parkinson's Disease. International journal of molecular sciences, 2017, 18(1), 224.

13. R.H. Gonzalez, M. Hamblin \& J.P. Lee. Neural stem cell transplantation and cns diseases. CNS \& Neurological Disorders-Drug Targets (Formerly Current Drug Targets-CNS \& Neurological Disorders), 2016, 15(8). 
14. A. Vogel, R. Upadhya, A.K. Shetty. Neural stem cell derived extracellular vesicles: Attributes and prospects for treating neurodegenerative disorders. EBioMedicine, 2018.

15. Y. Tang, P. Yu, L. Cheng. Current progress in the derivation and therapeutic application of neural stem cells. Cell Death Dis. 2017, 8(10): e3108. Published 2017 Oct 12. doi:10.1038/cddis.2017.504 